\title{
Classical transport of charged particles in a magnetic field
}

Noel Corngold ${ }^{\text {a) }}$

Watson Laboratories, California Institute of Technology, Pasadena, California 91125

(Received 8 September 2002; accepted 23 April 2003)

\begin{abstract}
We examine the traditional transport equation for classical, charged particles diffusing in a cold, absorbing medium subject to a uniform magnetic field and in which scattering is isotropic. Steady-state solutions in plane geometry are examined in some detail; we make no expansion about an isotropic angular distribution. Restricting the motion to two dimensions captures most of the interesting features; there is some discussion of the three-dimensional case. (C) 2003 American Institute of Physics. [DOI: 10.1063/1.1591994]
\end{abstract}

\section{INTRODUCTION}

The motion of ions and electrons in a weakly ionized gas subjected to external electric and magnetic fields is an ancient and "classical" problem in kinetic theory. ${ }^{1,2}$ The analysis of these systems ("swarms") is based, almost always, upon a linear "Boltzmann," or transport equation. The equation is treated in its most general form so that one can be as faithful as possible to the experiments. Most often the velocity variation of the distribution function is expressed in terms of a series of tensorial spherical harmonics, and the evolution of the density described in "hydrodynamic" and "nonhydrodynamic" terms. (Here, the interested reader should consult the impressive publications of Australian physicists. ${ }^{3-6}$ ) Such expansions-which may be viewed as a generalization of the traditional, Chapman-Enskog treatment of Boltzmann's equation-generate an infinite hierarchy of "moment-equations," a hierarchy which is truncated at some level, for reasons which are physical or practical. This procedure, with its advantages and its limitations, has been part of transport theory almost since its creation. Since these expansions are usually asymptotic, at best, one welcomes comparison with exact solutions of related transport equations which are not trivial.

This paper is concerned with the solution of such a relevant model-it describes the timeindependent transport of charged test-particles in a cold medium which scatters isotropically, may capture particles, and is immersed in a uniform magnetic field. Since no electric field is present, the distribution in velocity is simpler, but still interesting. In the solution, the most important dimensionless parameter is $\omega$, the ratio of cyclotron frequency to collision frequency. If one wishes to go beyond treating the analysis as a mathematical exercise, merely, one notes that $\omega$ is trivially small for ions in realistic situations, but can be interestingly large for electrons. Then, the (asymptotic) velocity distribution deviates considerably from the isotropic form about which the simplest "hydrodynamic" models are centered. Other results are-apart from an array of attractive equations involving Bessel functions-(1) that the magnetic field shatters the famous continuous spectrum associated with the "one-speed transport equation" " into an infinity of discrete eigenvalues. (We discuss the behavior of these eigenvalues as field and absorption strength are varied; the limit $\omega \rightarrow 0$ is quite singular.) (2) The magnetic field induces a flow parallel to the source plane. This "diamagnetic drift" is well known to plasma physicists. ${ }^{8}$ Here we treat it in some detail. Most important is that none of our results rely upon an assumption of "small gradients."

Justifying the irreversible, transport-rather than the reversible, Liouville treatment of a problem in kinetic theory-poses famous and delicate problems. In the case of an isolated and simple gas one justifies mathematically the transition from Liouville to transport by a limiting

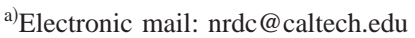


process-by invoking the "Grad-limit." "To the physicist, this means, roughly, that the gas should be sufficiently dilute for transport theory to be useful. When the system is subject to external fields, matters become more difficult. Recently, a group of scholars ${ }^{10}$ has analyzed the limiting process for the Lorentz model - which describes charged particles diffusing through a static array of scatterers-immersed in a magnetic field. Their analysis considers how unusual trajectories-a particle whirling in circular orbit in the space between two scatterers, for example-are to be treated in the construction of the "correct," coarse-grained transport equation. Our paper avoids these difficult issues. We simply study the "traditional" transport equation as a mathematical object, acknowledging that it describes the physics correctly in some regime where the magnetic field is not too strong and that often, continuing results obtained from a sanctioned region, say $\omega<1$, into a questionable one, say $\omega \gg 1$, has some value.

Most of the paper is devoted to motion in two dimensions (2D) - in a plane perpendicular to the uniform magnetic field. We give, briefly, expressions for densities and currents in the threedimensional case as well. In treating the equation, its features and consequences, we allow the strength of the uniform magnetic field to vary arbitrarily. The fact that a steady source of particles in an absorbing medium produces a steady distribution indicates that the traditional equation gives no weight to particles whirling, collisionless in tight circles,

\section{ANALYSIS OF A SIMPLE MODEL}

We begin with a simple model: noninteracting charged particles of fixed kinetic energy move in a plane perpendicular to an uniform magnetic field. The particles scatter elastically and isotropically from host atoms ("neutrals.") We are in steady state, there is a plane source ( $y-z$ plane) at the origin, and the medium, which is unbounded, may absorb particles. The distribution function for position and velocity, $F(x, \phi)$, depends then upon two variables, and we face the kinetic equation

$$
\mu \frac{\partial}{\partial x} F(x, \phi)-\omega \frac{\partial}{\partial \phi} F(x, \phi)=\frac{c}{2 \pi} \int_{0}^{2 \pi} \mathrm{d} \phi F(x, \phi)-F(x, \phi)+Q(\phi) \delta(x) .
$$

This familiar equation, with $(v \cdot x)=\mu=\cos \phi$, is written in dimensionless variables. The constant speed is taken to be unity, distance is scaled by the total mean-free-path for encounters, time by the corresponding mean-free-time, and $\omega$ represents the cyclotron frequency $q B / m c$ divided by the collision frequency. $\omega$ may also be seen as the ratio of mean-free-path to Larmor radius. $c$, the number of secondaries produced per collision, is limited to $1>c \geqslant 0$. As we see later, transport in three dimensions-with plane symmetry-is not that much more difficult.

After Fourier transformation in $x$ we have

$$
\mathcal{O} f(k, \phi)=-\omega \frac{\partial}{\partial \phi} f(k, \phi)+(1+i k \mu) f(k, \phi)=\frac{c}{2 \pi} \int_{0}^{2 \pi} \mathrm{d} \phi f(k, \phi)+Q(\phi)
$$

or

$$
(\mathcal{O}-c \mathcal{P}) f(k, \phi)=Q(\phi) .
$$

A new notation, Dirac-like, helps. Our operators act in function spaces whose elements are distribution functions $|f\rangle$. and $\langle g|$. There is a complex inner product. Thus,

$$
\langle g \mid f\rangle \equiv \int_{0}^{2 \pi} \mathrm{d} \phi g^{*}(\phi) f(\phi)=\langle f \mid g\rangle^{*}
$$

and

$$
(\mathcal{O}-c \mathcal{P})|f\rangle=|Q\rangle
$$


$\mathcal{P}$ is the projection operator,

$$
\mathcal{P}|f\rangle \equiv|a\rangle\langle a \mid f\rangle, \quad\langle a \mid a\rangle=1
$$

with

$$
|a\rangle=\frac{1}{\sqrt{2} \pi}, \quad\langle a \mid f\rangle=\int_{0}^{2 \pi} \mathrm{d} \phi \frac{1}{\sqrt{ } 2 \pi} f(\phi) .
$$

We may also use $|1\rangle=\sqrt{ } 2 \pi|a\rangle$, so that $\langle 1 \mid f\rangle=\int_{0}^{2 \pi} \mathrm{d} \phi f(k, \phi)$ gives $n(k)$, the angle-integrated density. The particle currents are

$$
\mathcal{J}_{x, y}(k)=\int_{0}^{2 \pi} \mathrm{d} \phi[\cos \phi, \sin \phi] f(k, \phi) .
$$

We use the special notation $\mathcal{J}_{x, y}(k)=\left\langle j_{x, y} \mid f\right\rangle$ for the currents.

Two results follow quickly; the solution to the kinetic equation, Eq. (3), is

$$
|f\rangle=\frac{1}{\mathcal{O}}|Q\rangle+c \frac{1}{\mathcal{O}}|a\rangle \frac{\left\langle a\left|\frac{1}{\mathcal{O}}\right| Q\right\rangle}{\left[1-c\left\langle a\left|\frac{1}{\mathcal{O}}\right| a\right\rangle\right]},
$$

and if we take the particularly simple case of an isotropic source of strength $|Q\rangle=|a\rangle$ we find

$$
\begin{gathered}
|f\rangle=\frac{1}{\left[1-c\left\langle a\left|\frac{1}{\mathcal{O}}\right| a\right\rangle\right]} \frac{1}{\mathcal{O}}|a\rangle=\frac{1}{\mathcal{D}(k, c)} \frac{1}{\mathcal{O}}|a\rangle, \\
\langle a \mid f\rangle=\frac{1}{\left[1-c\left\langle a\left|\frac{1}{\mathcal{O}}\right| a\right\rangle\right]}\left\langle a\left|\frac{1}{\mathcal{O}}\right| a\right\rangle=\frac{1}{\mathcal{D}(k, c)}\left\langle a\left|\frac{1}{\mathcal{O}}\right| a\right\rangle .
\end{gathered}
$$

The last equation also states that with unit source, $\left|Q_{1}\right\rangle=1 / 2 \pi$,

$$
n(k)=\frac{1}{\mathcal{D}(k, c)}\left\langle a\left|\frac{1}{\mathcal{O}}\right| a\right\rangle=\frac{\mathcal{K}(k)}{\mathcal{D}(k, c)}=\frac{\mathcal{K}(k)}{1-c \mathcal{K}(k)} .
$$

There are corresponding expressions for the currents,

$$
\mathcal{J}_{x, y}(k)=\frac{1}{\mathcal{D}(k, c)}\left\langle j_{x, y}\left|\frac{1}{\mathcal{O}}\right| a\right\rangle
$$

for source $|Q\rangle=|a\rangle$.

An alternative expression for $\mathcal{J}_{x}(k)$ appears if we integrate Eq. (1) directly, to get the equation of continuity,

$$
\frac{\partial}{\partial x} \mathcal{J}_{x}(x)+(1-c) n(x)=Q \delta(x) .
$$

We find 


$$
i k \mathcal{J}_{x}(k, c)=\frac{1-\mathcal{K}(k)}{1-c \mathcal{K}(k)}=\frac{\mathcal{D}(k, 1)}{\mathcal{D}(k, c)}
$$

for unit source $\left|Q_{1}\right\rangle$. That the various expressions are related by partial integration will be evident later, as will the expression

$$
i \mathcal{J}_{y}(k, c)=\frac{\omega}{2} \frac{1}{\mathcal{D}(k, c)} \frac{\partial}{\partial k} \mathcal{K}(k)
$$

The quantity

$$
\mathcal{K}(k)=\left\langle a\left|\frac{1}{\mathcal{O}}\right| a\right\rangle
$$

contains the essence of the system, generating the relaxation lengths, for example. This kernel is the Fourier transform of the kernel controlling the Peierls integral equation ${ }^{11}$ for $n(x)$ (see the following). We shall refer to $\mathcal{D}(k, c)=1-c \mathcal{K}$ as the dispersion function. This article is concerned with the features of density and current. To get at them, we learn as much as we can about $\mathcal{K}(k)$ and $\mathcal{D}(k, c)$.

\section{A. Evaluating the kernel}

Of the several ways to evaluate the kernel; two are particularly helpful. In the first, we make use of the eigen-vectors of the operator $\mathcal{O}$,

$$
\mathcal{O}|n\rangle=\lambda_{n}|n\rangle
$$

One finds

$$
\begin{gathered}
|n\rangle=\frac{1}{\sqrt{2 \pi}} \exp [i n \phi] \exp \left[i \frac{k}{\omega} \sin \phi\right], \\
\lambda_{n}=1-i n \omega .
\end{gathered}
$$

The $|n\rangle$ are complete and orthonormal, with the usual complex inner product. The wave number $k$ appears parametrically in the eigenvector but not in the eigenvalue. The $|n\rangle$ form a useful basis for expansion. Since

$$
\langle a \mid n\rangle=\frac{1}{2 \pi} \int_{0}^{2 \pi} \mathrm{d} \phi \exp [i n \phi] \exp \left[i \frac{k}{\omega} \sin \phi\right]=(-)^{n} J_{n}\left(\frac{k}{\omega}\right)=\langle n \mid a\rangle .
$$

Bessel functions make their expected appearance. A quite straightforward calculation leads then to the first of several expressions for the kernel,

$$
\left\langle a\left|\frac{1}{\mathcal{O}}\right| a\right\rangle=\left[J_{0}\left(\frac{k}{\omega}\right)\right]^{2}+2 \sum_{n=1}^{\infty}\left[J_{n}\left(\frac{k}{\omega}\right)\right]^{2} /\left(1+n^{2} \omega^{2}\right) .
$$

(In the following text, we may denote $k / \omega$ by $\kappa$, which is proportional to the ratio of cyclotron radius to wavelength of the $k$ mode.)

Another straightforward calculation, making use of $\mathcal{J}_{ \pm}=\mathcal{J}_{x} \pm i \mathcal{J}_{y}$ and recursion relations, leads to Eq. (7) for the transverse current - the "diamagnetic drift" of the plasma physicists. We note an alternate derivation ahead.

A more powerful and elastic representation of the kernel stems from an "algebraic" method, which is a simplified version of the method of characteristics. 
Write

$$
\begin{gathered}
\frac{1}{\mathcal{O}}|f\rangle=\int_{0}^{\infty} \mathrm{d} t e^{-t} e^{-t(A+B)}|f\rangle, \\
(A+B)=i k \cos \phi-\omega \frac{\partial}{\partial \phi}=e^{i \kappa \sin \phi}\left(-\omega \frac{\partial}{\partial \phi}\right) e^{-i \kappa \sin \phi} .
\end{gathered}
$$

Multiplication by $t$ and constructing the exponential series gives

$$
e^{-t(A+B)}=e^{i \kappa \sin \phi} \exp \left(\omega t \frac{\partial}{\partial \phi}\right) e^{-i \kappa \sin \phi},
$$

and we can write, quite generally,

$$
\left\langle g\left|\frac{1}{\mathcal{O}}\right| Q\right\rangle=\int_{0}^{\infty} \mathrm{d} t e^{-t} \int_{0}^{2 \pi} \mathrm{d} \phi g(\phi)^{*} e^{i \kappa[\sin \phi-\sin (\phi+\omega t)]} Q(\phi+\omega t) .
$$

In particular, our kernel is

$$
\left\langle a\left|\frac{1}{\mathcal{O}}\right| a\right\rangle=\frac{1}{2 \pi} \int_{0}^{\infty} \mathrm{d} t e^{-t} \int_{0}^{2 \pi} \mathrm{d} \phi e^{i \kappa[\sin \phi-\sin (\phi+\omega t)]},
$$

and inversion of the Fourier transform gives the Peierls integral equation which corresponds to Eq. (1). One finds-for an isotropic source-

$$
n(x)=\frac{1}{2 \pi} \int_{-\infty}^{\infty} \mathrm{d} x^{\prime} \mathcal{K}\left(x-x^{\prime}\right)\left[n\left(x^{\prime}\right)+q\left(x^{\prime}\right)\right]
$$

with

$$
\mathcal{K}\left(x-x^{\prime}\right)=\int_{0}^{\infty} \mathrm{d} t e^{-t} \int_{0}^{2 \pi} \mathrm{d} \phi \delta\left(x-x^{\prime}+[\sin \phi-\sin (\phi+\omega t)]\right) .
$$

After some reduction, using the fact that the integral of a periodic function over its period is unaltered by a shift in the variable of integration, we find

$$
\mathcal{K}\left(x-x^{\prime}\right)=\frac{1}{1-e^{-4 \pi / \omega}} \int_{0}^{2 \pi} \mathrm{d} \theta \int_{0}^{2 \pi} \mathrm{d} \phi e^{-2 \theta / \omega} \delta\left(\frac{\omega}{2}\left(x-x^{\prime}\right)-\sin \theta \sin \phi\right) .
$$

$\mathcal{K}$ is even, in its argument. In our dimensionless units $\omega$, the ratio of cyclotron frequency to collision frequency is the reciprocal of the ratio of cyclotron radius to mean-free-path. Thus, the constraint expressed by the $\delta$-function, that $\left|x-x^{\prime}\right| \leqslant 2 / \omega$, is precisely the statement that the farthest-ranging particle is found at one "cyclotron-diameter" from a plane source, having left the source traveling parallel to it. The Peierls' kernel, $\mathcal{K}(x)$, has "compact support"; it has no exponential tail. Further reduction will be remarked upon later. [See Eq. (24).]

Returning to the kernel, note the limit $\omega \rightarrow 0$ when

$$
\left\langle a\left|\frac{1}{\mathcal{O}}\right| a\right\rangle \rightarrow \frac{1}{2 \pi} \int_{0}^{2 \pi} \mathrm{d} \phi \frac{1}{1+i k \cos \phi}=\frac{1}{\sqrt{1+k^{2}}} .
$$


In the field-free case, the kernel and the dispersion function are analytic in a cut, complex- $k$ plane, and spatial relaxation is described by a discrete and a continuous spectrum of relaxation lengths. The Fourier inverse of the kernel,

$$
\frac{1}{2 \pi} \int_{-\infty}^{\infty} \mathrm{d} k e^{i k x}\left\langle a\left|\frac{1}{\mathcal{O}}\right| a\right\rangle=\frac{1}{\pi} K_{0}(|x|),
$$

expresses the density of uncollided particles as a Bessel function. Another useful limiting expression holds when $\omega \gg 1$. Then Eq. (10) informs us that

$$
\left\langle a\left|\frac{1}{\mathcal{O}}\right| a\right\rangle \rightarrow\left[J_{0}\left(\frac{k}{\omega}\right)\right]^{2} .
$$

The kernel, as expressed by Eq. (13), may be simplified if we expand the exponential, noting that only even powers survive the angle-averaging. One finds that when $n=2 m$,

$$
\frac{1}{2 \pi} \int_{0}^{2 \pi} \mathrm{d} \phi[\sin \phi-\sin (\phi+\omega t)]^{n}=\left(\begin{array}{c}
2 m \\
m
\end{array}\right)\left[\sin \left(\frac{\omega t}{2}\right)\right]^{2 m} .
$$

Now, two paths are open; one can use

$$
\frac{1}{(2 m) !} \int_{0}^{\infty} \mathrm{d} t e^{-t}\left(\sin \frac{\omega}{2} t\right)^{2 m}=\omega^{2 m} \prod_{r=1}^{m} \frac{1}{1+r^{2} \omega^{2}},
$$

to get the useful series

$$
\left\langle a\left|\frac{1}{\mathcal{O}}\right| a\right\rangle=1+\sum_{m=1}^{\infty}(-)^{m}\left(\begin{array}{c}
2 m \\
m
\end{array}\right)\left(\frac{k^{2}}{4}\right)^{m} \prod_{r=1}^{m} \frac{1}{1+r^{2} \omega^{2}},
$$

or eschew the $t$-integration, recognizing the series as generating the Bessel function $J_{0}$, and hence that

$$
\mathcal{K}(k)=\left\langle a\left|\frac{1}{\mathcal{O}}\right| a\right\rangle=\int_{0}^{\infty} \mathrm{d} t e^{-t} J_{0}\left(2 \frac{k}{\omega} \sin \frac{\omega t}{2}\right) .
$$

(The Appendix contains a shorter derivation of this result.)

Equation (17) may be rearranged by exploiting periodicity, and using

$$
\int_{0}^{\pi} \mathrm{d} \phi g(\phi) F(\sin \phi)=\int_{0}^{\pi / 2} \mathrm{~d} \phi\left[g\left(\frac{\pi}{2}-\phi\right)+g\left(\frac{\pi}{2}+\phi\right)\right] F(\cos \phi),
$$

to get

$$
\begin{aligned}
\mathcal{K}(k) & =\frac{2}{\omega} \frac{1}{1-e^{-2 \pi / \omega}} \int_{0}^{\pi} \mathrm{d} \phi e^{-2 \phi / \omega} J_{0}\left(2 \frac{k}{\omega} \sin \phi\right) \\
& =\frac{2}{\omega} \frac{1}{\sinh \left(\frac{\pi}{\omega}\right)} \int_{0}^{\pi / 2} \mathrm{~d} \phi \cosh \left(\frac{2 \phi}{\omega}\right) J_{0}\left(2 \frac{k}{\omega} \cos \phi\right),
\end{aligned}
$$

all of which have been found useful. A nice connection with the eigenfunction expansion, Eq. (10), is made via Neumann's addition formula 


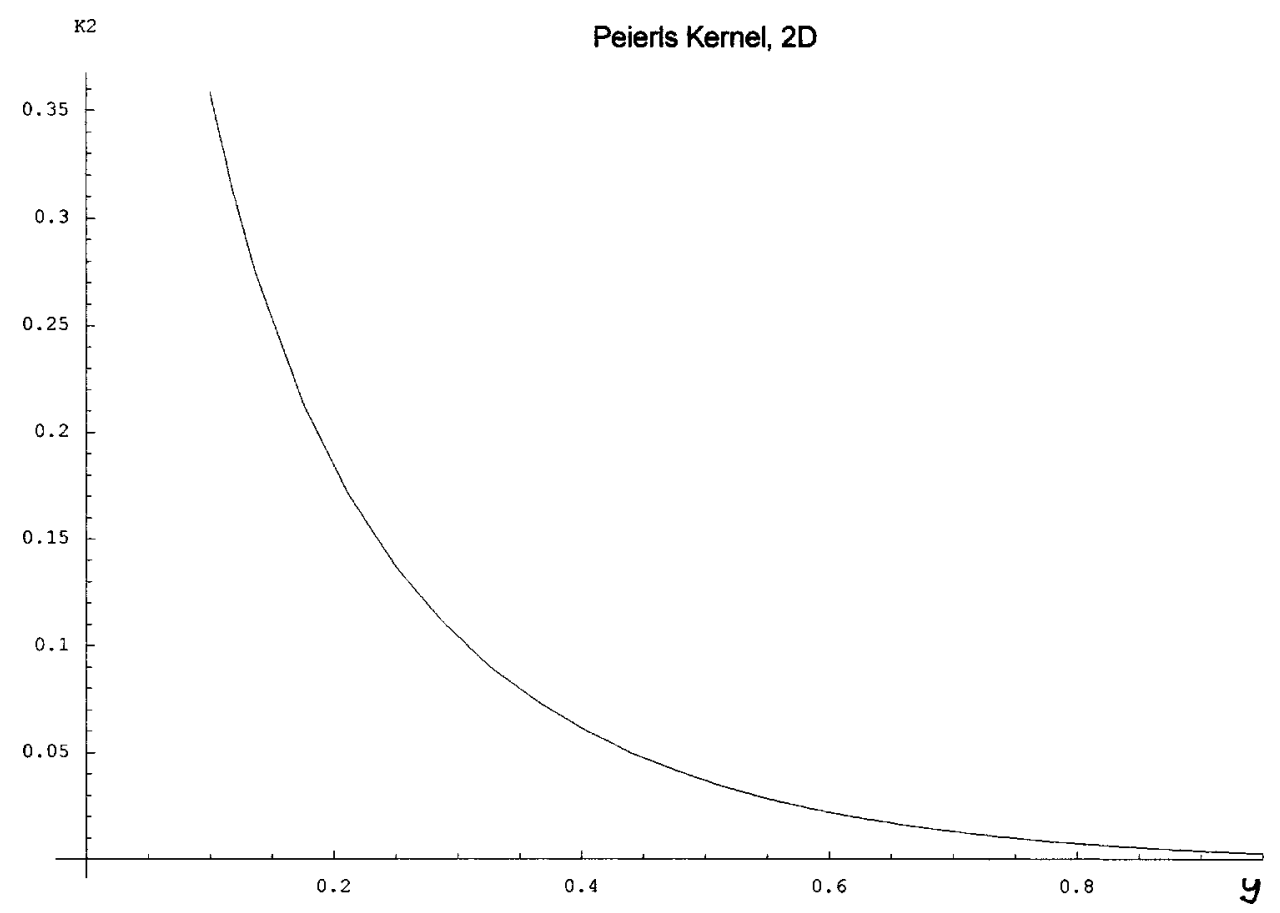

FIG. 1. $\mathcal{K}_{2}$, the 2D kernel (Peierls) for the integral equation for density, $n(x)$, with $\omega=0.5$, shown as a function of its argument, $y=\omega|x| / 2$. The kernel is zero when $y>1$ while $\mathcal{K}_{2}\left(1^{-}\right)=1 /[2 \sinh (\pi / \omega)]$.

$$
J_{0}(2 t \sin \psi)=J_{0}(t)^{2}+2 \sum_{n=1}^{\infty} J_{n}(t)^{2} \cos 2 n \psi
$$

in concert with Eq. (17).

Finally, we may Fourier-invert Eq. (19) to get another representation of the Peierls kernel, namely

$$
\begin{aligned}
\mathcal{K}(x) & =\frac{1}{\pi} \frac{1}{\sinh \left(\frac{\pi}{\omega}\right)} \int_{0}^{\phi_{*}(\omega x)} \mathrm{d} \phi \frac{\cosh \left(\frac{2 \phi}{\omega}\right)}{\sqrt{ } \cos ^{2} \phi-\cos ^{2} \phi_{*}(\omega x)}, \quad 0<x<\frac{2}{\omega} \\
& =0, \quad x>\frac{2}{\omega},
\end{aligned}
$$

where $\phi_{*}$ is defined through $\cos \phi_{*}=(\omega / 2) x$. (See Fig. 1.)

A final comment for this section: There is still another way to solve our transport equation, Fourier expansion in $\phi$. That approach, which is more natural when the scattering is anisotropic, leads to difference equations, to solutions in terms of continued fractions and to the traditional, truncated solutions which we wish to avoid.

\section{B. The complex $k$ plane}

The Fourier inversion of $\langle a \mid f\rangle$ and $\left\langle j_{x, y} \mid f\right\rangle$ will be controlled by singularities in the complex $k$ plane. The series in Eq. (16) converges throughout the $k$ plane for all nonzero $\omega$, assuring us that our kernel is an entire function of $k$. Thus, the only singularities are the zeros of the dispersion function. The magnetic field has shattered the continuous spectrum and it is to the zeros that we turn. They will describe the relaxation lengths which characterize the system. Since their location 
depends upon both magnetic field and absorption, $0 \leqslant \omega<\infty$ and $0 \leqslant c \leqslant 1$, the picture, overall, is complicated. But the analysis is eased somewhat by the fact that $c$ and $\omega$ occur separately in the dispersion function. We begin by observing the following.

(1) Since $\mathcal{D}$ is a real function of $k^{2}$, a complex root of the dispersion equation will generate four zeros in the $k$ plane, of the form $\pm(\xi \pm i \eta)$. Thus we may limit our discussion to a single quadrant of the complex $k$ plane, with the understanding that the other three will be filled by reflection.

(2) If the particle distribution is to be bounded at infinity we expect that $\mathcal{D}$ has no zeros on the real axis of $k$. (The exceptional case $c=1$ produces a second-order zero at the origin, and is "physical" when the source is moved to infinity-the Milne case.) And inspection of the series for $\mathcal{D}$ shows that when $c=1$, there are no zeros (poles) on the imaginary axis.

(3) The dominant zero. When $0<c<1$ there is a dominant zero (pole) on the positive (and the negative) sections of the imaginary axis, where $k=i \eta$. This pole, with its associated residue, describes the dominant, large- $x$, "asymptotic" behavior of the distribution. There is no other zero on the axis because the kernel is a function of $\eta$ which increases smoothly. (The zeros which are off-axis and are discussed later, give oscillatory contributions which cannot dominate, asymptotically.) Then, for the dominant zero, we have four regimes as follows.

(i) Strong field ( $\omega \gg 1, c$ arbitrary). Here, Eq. (15) enables us to write

$$
\eta_{0}=\omega y(c),
$$

where

$$
1-c I_{0}(y)^{2}=0
$$

for all $c$. (In practice, the estimate is valuable even at $\omega \approx 1$.)

(ii) Weak absorption $(1-c \ll 1, \omega$ arbitrary). Here, the zero lies close to the origin, and the series, Eq. (16) yields

$$
\eta_{0} \simeq \sqrt{2(1-c)\left(1+\omega^{2}\right)},
$$

a result which emerges from Eq. (21), too. It describes a simple, exponential attenuation, which has been increased by the magnetic field. In the field-free case $\eta_{0}=\sqrt{1-c^{2}}$ exactly.

(iii) Strong absorption ( $c \rightarrow 0, \omega$ arbitrary). When field is absent the kernel displays branch points, and the zero falls into the branch singularity when $c=0$. With field present, there are no branch points and matters are quite different. The dominant zero moves along the imaginary axis to infinity as $c \rightarrow 0$, the dominant relaxation length becoming arbitrarily small. It is the magnetic field, rather than the mean free-path, which is in control. Equation (21) yields an easy estimate, when the field is large,

$$
\eta_{0} \simeq \frac{\omega}{2} \log \left(\frac{1}{c}\right) .
$$

In the limit, $c=0$, the distribution becomes that of uncollided particles proceeding from a plane, isotropic source. It is simply the Peierls kernel, Eq. (20). It helps to write the symmetric distribution as $(x>0)$ 


$$
\begin{aligned}
& n_{0}(x)=\mathcal{K}(x)=\frac{1}{\pi} \frac{\cosh \left(\frac{2 \phi_{*}(\omega x)}{\omega}\right)}{\sinh \left(\frac{\pi}{\omega}\right)} \int_{0}^{\phi_{*}(\omega x)} \mathrm{d} \phi \frac{1}{\sqrt{ } \cos ^{2} \phi-\cos ^{2} \phi_{*}(\omega x)} \\
& +\frac{1}{\pi} \frac{1}{\sinh \left(\frac{\pi}{\omega}\right)} \int_{0}^{\phi_{*}(\omega x)} \mathrm{d} \phi \frac{\cosh \left(\frac{2 \phi}{\omega}\right)-\cosh \left(\frac{2 \phi_{*}(\omega x)}{\omega}\right)}{\sqrt{ } \cos ^{2} \phi-\cos ^{2} \phi_{*}(\omega x)}, \quad 0<x<\frac{2}{\omega} \\
& =0, \quad x>\frac{2}{\omega} .
\end{aligned}
$$

The key quantity in its description is $\phi_{*}(\omega x)$, which descends from $(\pi / 2)$ at the plane source to zero at the edge of the distribution. We note further that (a) the second term in Eq. (24) is bounded for all $\phi_{*}(x)$, vanishing at the edge of the distribution, while (b) the integral appearing in the first term may be transformed into the complete elliptical integral $K\left(\sin ^{2} \phi_{*}\right)$. It diverges, logarithmically at the source plane, and is $(\pi / 2)$ at the edge. Thus, the distribution of uncollided particles is discontinuous at its edge. We have the limits (see Fig. 1)

$$
\begin{gathered}
n_{0}(x)=\mathcal{K}(x) \rightarrow \frac{1}{2 \sinh \left(\frac{\pi}{\omega}\right)}, \quad x \rightarrow \frac{2}{\omega}, \\
n_{0}(x)=\mathcal{K}(x) \sim \frac{1}{\pi} \operatorname{coth}\left(\frac{\pi}{\omega}\right) \log \left(\frac{1}{x}\right)+\cdots, \quad x \rightarrow 0 .
\end{gathered}
$$

The size of the step-discontinuity vanishes, conveniently, as $\omega \rightarrow 0$, while the leading behavior at the source plane coincides with that of the field-free case.

(iv) Weak field ( $\omega \rightarrow 0, c$ arbitrary). While the large- $\omega$ limit is relatively simple, the small- $\omega$ limit is not. Since singularities which are absent when $\omega \neq 0$ appear (at $\eta= \pm 1$ ) when $\omega=0$, the behavior in the neighborhood of these points cannot be analytic. The simple expression, Eq. (22), does not tell the full story. We begin by studying

$$
\mathcal{I}(\phi, \eta)=\cosh \left(\frac{2}{\omega} \phi\right) I_{0}\left(\frac{2}{\omega} \eta \cos \phi\right)
$$

the integrand appearing in Eq. (19), in the regime $(2 / \omega) \equiv \lambda \gg 1$. At the limits of integration we have

$$
\mathcal{I}(0, \eta)=I_{0}(\lambda \eta), \quad \mathcal{I}\left(\frac{\pi}{2}, \eta\right)=\cosh \left(\lambda \frac{\pi}{2}\right)
$$

and behavior will be different in different intervals of $\eta$, for absorption may cause $\eta_{0}$ to be large. It is no surprise that $\eta=1$, and $\eta=\pi / 2$, play important roles in the analysis.

(a) When $\eta<1, \mathcal{I}(\phi, \eta)$ is seen to be an increasing function of $\phi$ in $(0, \pi / 2)$ and the integral is controlled by the behavior of $\mathcal{I}$ near its maximum —at the edge. If we rearrange Eq. (19) to place the maximum at the origin we obtain

$$
\mathcal{K}(i \eta)=\frac{1}{2} \int_{0}^{\Lambda} \mathrm{d} t I_{0}\left(\lambda \eta \sin \left(\frac{t}{\lambda}\right)\right)\left\{(\operatorname{coth}(\Lambda)-1) e^{t}+(\operatorname{coth}(\Lambda)+1) e^{-t}\right\},
$$

where 
TABLE I. $1 / \eta_{0}$ is the relaxation length, in units of mean-free-path, for the particle distribution far from its plane source, in the presence of various amounts of capture and various magnetic field strengths.

\begin{tabular}{lccccc}
\hline \hline & \multicolumn{5}{c}{ Dominant zero, $\eta_{0}$} \\
\cline { 2 - 6 }$\omega$ & $c=0.9$ & $c=0.7$ & $c=0.5$ & $c=0.3$ & $c=0.1$ \\
\hline 0.25 & 0.46 & 0.78 & 1.0 & 1.2 & 1.5 \\
0.5 & 0.50 & 0.89 & 1.2 & 1.5 & 2.0 \\
1 & 0.65 & 1.2 & 1.6 & 2.1 & 3.0 \\
\hline \hline
\end{tabular}

$$
\Lambda=\frac{\pi}{\omega}=\frac{\pi}{2} \lambda .
$$

A little consideration shows that when $\lambda$ is very large we may write

$$
\mathcal{K}(i \eta) \simeq \int_{0}^{\infty} \mathrm{d} t I_{0}\left(\lambda \eta \sin \left(\frac{t}{\lambda}\right)\right) e^{-t}
$$

with exponentially small error, $O\left(e^{-(1-\eta) \Lambda}\right)$. Then, expansion about $t=0$, aided by the relation

$$
\frac{1}{n !} \int_{0}^{\infty} \mathrm{d} t e^{-t} t^{n} J_{0}(k t)=\frac{1}{\left(1+k^{2}\right)^{(n+1 / 2)}} P_{n}\left(\frac{1}{\sqrt{1+k^{2}}}\right)
$$

( $P_{n}$ is the Legendre polynomial) yields

$$
\mathcal{K}(i \eta)=\left\langle a\left|\frac{1}{\mathcal{O}}\right| a\right\rangle=\frac{1}{\sqrt{ } 1-\eta^{2}}\left[1-\frac{1}{2}\left(\frac{\omega}{2}\right)^{2} \eta^{2} \frac{4+\eta^{2}}{\left(1-\eta^{2}\right)^{3}}+\cdots\right],
$$

in which the singular nature of the small- $\omega$ behavior is displayed.

In the complimentary situation, when $\eta>1, \mathcal{I}(\phi, \eta)$ posesses a maximum inside the interval $(0, \pi / 2)$. When that sharp maximum dominates the contribution from the edge, expansion produces

$$
\mathcal{K}(i \eta) \simeq \frac{1}{\sqrt{\eta^{2}-1}} \exp \left[\left(\frac{2}{\omega}\right)\left(\sin ^{-1} \frac{1}{\eta}-\frac{\pi}{2}+\sqrt{\eta^{2}-1}\right)\right],
$$

as $(2 / \omega) \rightarrow \infty$. Thus, when $\eta \gg 1$ as well,

$$
\mathcal{K}(\text { i } \eta) \simeq \frac{1}{\eta} \exp \left[\frac{2}{\omega}\left(\eta-\frac{\pi}{2}\right)\right],
$$

which leads easily to the estimate

$$
\eta_{0} \simeq \frac{\omega}{2} \log \left(\frac{1}{c}\right) \quad \text { for } c \rightarrow 0 \text { and } \omega \text { "small," }
$$

familiar, and in agreement with Eq. (23).

Some dominant zeros are displayed in Table I.

(4) Transients. The other zeros, or relaxation lengths, form a complex pattern. They produce transient, oscillating terms in the spatial distribution. We would like a picture of the pattern, and how it alters as the magnetic field is altered. In particular, we are interested in the limit $\omega \rightarrow 0$. Ultimately, we rely on the summation, numerically, of Eq. (16). But the search for zeros is helped greatly by asymptotic estimates, to which we turn. 
(i) Strong field $(\omega \gg 1,|\kappa|$ arbitrary) $(\kappa \equiv k / \omega)$. We begin with the simplest estimate, $\omega \gg 1$, when a different approach yields a familiar result,

$$
\mathcal{K}(k) \simeq 1+\sum_{m=1}^{\infty}\left(\begin{array}{c}
2 m \\
m
\end{array}\right)\left(-\frac{k^{2}}{4 \omega^{2}}\right)^{m} \frac{1}{(m !)^{2}}=\frac{2}{\pi} \int_{0}^{\pi / 2} \mathrm{~d} \phi J_{0}\left(2 \frac{k}{\omega} \sin \phi\right)=J_{0}\left(\frac{k}{\omega}\right)^{2} .
$$

Then, the $\omega$ dependence is simple, $k_{n}=\omega \kappa_{n}$, where $\kappa_{n}(c)$ is one of the complex zeros of 1 $-c J_{0}(\kappa)^{2}$. (In practice, the estimate is valuable even at $\omega \approx 1$.) Among these, the "higher harmonics" $(|\kappa| \gg 1)$ obey

$$
\frac{\sin 2 x}{2 x} e^{2 y}=\frac{\pi}{c}, \quad \cos 2 x=2 y e^{-2 y} \frac{\pi}{c}
$$

where $\kappa=x+i y$. From these, we infer

$$
k_{n} \approx \omega\left\{n \pi+i \frac{1}{2} \log \left[\left(2 n+\frac{1}{2}\right) \frac{\pi^{2}}{c}\right]\right\}, \quad n=0,1,2, \ldots
$$

for the regime $\omega \gg 1$ and $|k| \gg \omega$. The real parts of the relaxation constants increase only logarithmically, the imaginary parts linearly. Note that the dominant zero is contained here, associated with $c \ll 1$, and behaves as

$$
k_{0} \simeq i \frac{\omega}{2} \log \left(\frac{1}{c}\right)
$$

(ii) Weak field is included in the regime $(|\kappa| \gg 1, \omega$ arbitrary). We begin with the estimate

$$
\int_{0}^{\pi} \mathrm{d} \phi e^{-2 \phi / \omega} J_{0}(2 \kappa \sin \phi) \sim \frac{1}{2 \kappa}\left[1+e^{-2 \pi / \omega}+2 e^{-\pi / \omega} \sin 2 \kappa\right], \quad|\kappa| \rightarrow \infty,
$$

derived by the method of stationary phase, including end-point corrections. The derivation proceeds with the assumption that $k$ is real, but suggests strongly that the result holds throughout the quadrant when $\operatorname{Re}(\kappa)>0$. This conjecture is supported by the fact that Eqs. (34) and (31) are identical, namely,

$$
\mathcal{K}(k) \approx \frac{i}{2 \pi \kappa} e^{-2 i \kappa}
$$

when their domains overlap, and by numerical experience. We have, then, the approximate dispersion equation,

$$
1+\left(\frac{2 e^{-\pi / \omega}}{1+e^{-2 \pi / \omega}}\right) \sin 2 \kappa=\frac{\kappa \omega}{c}\left(\frac{1-e^{-2 \pi / \omega}}{1+e^{-2 \pi / \omega}}\right)
$$

For field strengths $e^{-2 \pi / \omega} \ll 1$, and for $\kappa$ large, further simplification enables us to write the estimate as

$$
\begin{gathered}
c \xi=1+\exp \left[\frac{2}{\omega}\left(\eta-\frac{\pi}{2}\right)\right] \sin \frac{2 \xi}{\omega} \\
c \eta=\exp \left[\frac{2}{\omega}\left(\eta-\frac{\pi}{2}\right)\right] \cos \frac{2 \xi}{\omega}
\end{gathered}
$$


(recall that $k=\xi+i \eta$ ).

When $\omega$ is small these expressions are useful when $\eta>\pi / 2$. Then, with

$$
\eta=\frac{\pi}{2}+\frac{\omega}{2} \eta_{1}(\omega)
$$

and

$$
\xi=\frac{\omega}{2} \xi_{1}(\omega) .
$$

the new functions assumed to be regular, we are led to

$$
k_{n}=\omega\left[n \pi-\xi_{*}\right]+i\left[\frac{\pi}{2}+\frac{\omega}{4} \log \left\{1+\left(\frac{\pi c}{2}\right)^{2}\right\}\right]+o(\omega),
$$

where $\left(n \pi-\xi_{*}\right)=2 \xi_{1}(0)$ is the solution to

$$
\tan \left\{2 \xi_{1}(0)\right\}=-\frac{2}{\pi c}
$$

and $n=1,2, \ldots$ is not too large. This pattern of zeros, differing little in their imaginary parts, and marching into a point on the imaginary axis, is roughly ("semiquantitatively") correct. The numerical values it yields are helpful, even though the true point of accumulation is $k=i$ rather than $k=i(\pi / 2)$. Of course, the asymptotic expansion fails on the imaginary axis. There, and in an unknown strip containing it, we return to the expansion used in connection with Eq. (27) to obtain

$$
\mathcal{K}(k)=\left\langle a\left|\frac{1}{\mathcal{O}}\right| a\right\rangle=\frac{1}{\sqrt{k^{2}+1}}\left[1-\frac{1}{2}\left(\frac{\omega}{2}\right)^{2} k^{2} \frac{k^{2}-4}{\left(k^{2}+1\right)^{3}}+\cdots\right]
$$

a complicated series which exhibits clearly the branch-point singularity and omits exponentially small terms (in $\omega$ ). Before Eq. (37) is used to estimate zeros computation of the winding-number suggests that if we consider a small circular region about $k=i$, the number of zeros (poles) present in the region increases without limit as $\omega \rightarrow 0$. Analysis of the series suggests that the zeros do not follow distinct, ray-like paths as they fall into the (nascent) branch point. Rather, their paths merge. For example the first few terms of Eq. (37) yield a pair of roots and a merged path described by

$$
\begin{gathered}
k-i \approx \alpha \omega^{2 / 3} \exp \left(\frac{\pi}{6} i\right)\left[1 \mp \frac{1}{3} \sqrt{2 \alpha} \omega^{1 / 3} \exp \left(\frac{\pi}{3} i\right)\right], \\
\alpha=\frac{1}{4}(5)^{1 / 3}
\end{gathered}
$$

in the first quadrant. The dependence upon $\omega^{2 / 3}$ is borne out to a fraction of a percent by numerical calculation, and the $\pi / 6$, which characterizes the asymptote, appears to be correct, but the numerical coefficients need improvement. Overall the dependence upon $\omega$ is quite singular, for we have omitted exponentially singular factors. See Fig. 2. 


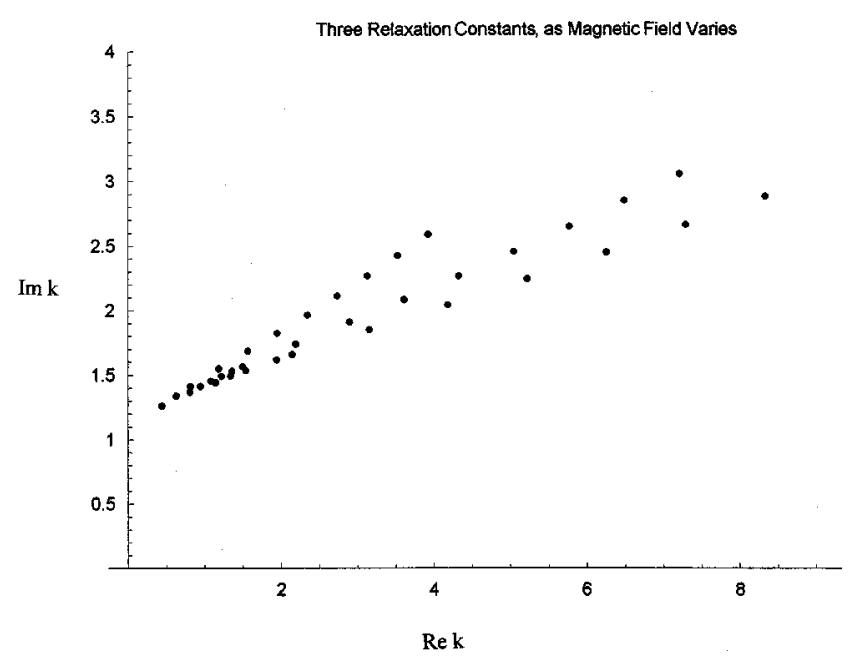

FIG. 2. Three zeros (poles) and their "motion" in the $k$ plane as the magnetic field $(\omega)$ is altered. $\omega \rightarrow 0$ brings them to $k=i$.

\section{Densities and currents}

The currents $\mathcal{J}_{x, y}(k)$ may be obtained from the kernel via Eqs. (6) and (7) or directly, from

$$
\frac{1}{\sqrt{2} \pi}\left\langle j_{ \pm}\left|\frac{1}{\mathcal{O}}\right| a\right\rangle=\int_{0}^{\infty} \mathrm{d} t e^{-t} \int_{0}^{2 \pi} \frac{\mathrm{d} \phi}{2 \pi} e^{ \pm i \phi} e^{i \kappa[\sin \phi-\sin (\phi+\omega t)]},
$$

which yields, for $\left|Q_{1}\right\rangle$ (unit source),

$$
\begin{aligned}
& i \mathcal{J}_{x}(k)=\frac{1}{\mathcal{D}(k, c)} \int_{0}^{\infty} \mathrm{d} t e^{-t} \cos \frac{\omega t}{2} J_{1}\left(2 \kappa \sin \frac{\omega t}{2}\right), \\
& -i \mathcal{J}_{y}(k)=\frac{1}{\mathcal{D}(k, c)} \int_{0}^{\infty} \mathrm{d} t e^{-t} \sin \frac{\omega t}{2} J_{1}\left(2 \kappa \sin \frac{\omega t}{2}\right),
\end{aligned}
$$

as symmetric alternatives to Eqs. (6) and (7). Also, there is Eq. (17),

$$
n(k)=\frac{1}{\mathcal{D}(k, c)} \int_{0}^{\infty} \mathrm{d} t e^{-t} J_{0}\left(2 \frac{k}{\omega} \sin \frac{\omega t}{2}\right) .
$$

Equations (6) and (7) follow easily from Eq. (39) by integration by parts.

Since $\mathcal{D}(k, c)$ is even in $k$, we conclude that the components of current change sign when $x$ is replaced by $-x$. Since $\mathcal{D}(k, c)$ diminishes with increasing $k$ [see Eq. (34)], the behavior of density and current near the source is linked to the behavior of the integrals in Eq. (39). These, which describe the uncollided particles, may be inverted and reduced to

$$
\begin{aligned}
\mathcal{J}_{0 x}(x) & =\frac{1}{\pi} \frac{\cos \phi_{*}(x)}{\sinh \left(\frac{\pi}{\omega}\right)} \int_{0}^{\phi_{*}(x)} \mathrm{d} \phi \sinh \left(\frac{2 \phi}{\omega}\right) \frac{\tan \phi}{\sqrt{\cos ^{2} \phi-\cos ^{2} \phi_{*}(x)}} \\
& =\frac{1}{2}-(1-c) x n_{0}(x)+\cdots,
\end{aligned}
$$




$$
\begin{aligned}
\mathcal{J}_{0 y}(x) & =-\frac{1}{\pi} \frac{\cos \phi_{*}(x)}{\sinh \left(\frac{\pi}{\omega}\right)} \int_{0}^{\phi_{*}(x)} \mathrm{d} \phi \frac{\cosh \left(\frac{2 \phi}{\omega}\right)}{\sqrt{\cos ^{2} \phi-\cos ^{2} \phi_{*}(x)}} \\
& =-\frac{\omega}{2} x n_{0}(x)
\end{aligned}
$$

near the source-plane. For the behavior ("asymptotic") far from the plane, we turn to inversion by contour integration to get

$$
\begin{gathered}
\mathcal{J}_{y}(x)=-\frac{\omega}{2 c} \sum_{n} e^{i k_{n} x}, \\
\mathcal{J}_{x}(x)=-\left(\frac{1-c}{c}\right) \sum_{n} \frac{1}{k_{n} D_{n}} e^{i k_{n} x}, \\
n(x)=\frac{i}{c} \sum_{n} \frac{1}{D_{n}} e^{i k_{n} x}
\end{gathered}
$$

with $D_{n} \equiv(\partial / \partial k) D\left(k_{n}, c\right)$. Though the summation is over all zeros in the upper half-plane, we are most interested in the contribution from the dominant zero. Note that the equation of continuity is satisfied, "mode by mode," and observe the strangely simple expression for $\mathcal{J}_{y}(x)$, as well as the singular behavior at $x=0$.

The transverse current, $\mathcal{J}_{y}(x)$, which is zero at the source plane, grows as one proceeds away. The ratio $\mathcal{J}_{y}(x) / \mathcal{J}_{x}(x)$ is then of some interest. The ratio assumes the value $\frac{1}{2}\left[k_{0} D_{0} /(1-c)\right] \omega$ asymptotically. One may compute the quantity easily in two limiting cases. In the first, very weak capture, $c \gg 1-c$, the ratio is simply $(-\omega)$. In the second, very high frequency, $\omega \gg 1$ [see Eqs. (16) and (31)] the ratio is $(-\omega \mathcal{F}(c))$, where

$$
\mathcal{F}(c)=\frac{\sqrt{c}}{1-c} \kappa_{0} J_{1}\left(\kappa_{0}\right), \quad J_{0}\left(\kappa_{0}\right)=\frac{1}{\sqrt{c}} .
$$

Diffusion: $\left(k_{0}=i \eta_{0}=\omega \kappa_{0}\right)$. The question of diffusion and diffusion constant may be viewed in two ways. One can note that far from the source the density follows $n_{x x}+k_{0}^{2} n=0$, an equation suggesting steady-state diffusion with diffusion coefficient

$$
\mathbb{D}_{x x}=\frac{(1-c)}{\eta_{0}^{2}} .
$$

More generally,

$$
\mathbb{D}_{x x}=\mathbb{D}_{y y}=\frac{(1-c)}{\eta_{0}^{2}}, \quad \mathbb{D}_{y x}=-\mathbb{D}_{x y}=\left.\frac{\omega}{2 k_{0}} \frac{\partial}{\partial k} \mathcal{D}(k, \omega)\right|_{k_{0}},
$$

the tensor components depending upon field strength and capture. This view is surely more helpful to the experimenter than is the traditional attitude that "Fick's law" holds throughout, that there is everywhere a proportionality between density gradient and current. In fact, the law holds only when spatial variations are so gentle ("long-ranged") that only the lowest powers in an expansion in $k$ need be retained. Then, Eq. (16) leads to 


$$
\left(\begin{array}{c}
\mathcal{J}_{x} \\
\mathcal{J}_{y}
\end{array}\right)=-\frac{1}{2}(i k) \frac{1}{1-c} \frac{1}{1+\omega^{2}}\left(\begin{array}{c}
1 \\
-\omega
\end{array}\right)
$$

describing "classical" anisotropic diffusion, with diffusion tensor

$$
\mathbb{D}_{i j}=\frac{1}{2} \frac{1}{1+\omega^{2}}\left(\begin{array}{cc}
1 & \omega \\
-\omega & 1
\end{array}\right)
$$

This simple picture, found in most textbooks, displays a "normal" diffusion, inhibited by the magnetic field, and transverse diffusion, induced by the field. The tensor multiplication may also be written

$$
\mathbb{D} \cdot \nabla n=\frac{1}{2} \frac{1}{1+\omega^{2}}[1-\omega \hat{z} \times] \nabla n .
$$

The two treatments differ little when capture is almost negligible and $k_{0}$ is small. The case of large field ("high frequency") is accessible through Eqs. (16) and (31) when Eq. (44) yields

$$
\mathbb{D}_{y x}=-\mathbb{D}_{x y}=-\frac{1}{\omega \sqrt{c}} \frac{J_{1}\left(\kappa_{0}\right)}{\kappa_{0}} .
$$

Thus, for fixed capture, the transverse diffusion is—again —inhibited by the magnetic field when the field is large. $D_{y x}$ rises, proportionally to $\omega$ when $\omega$ is small, reaches some peak value, then decreases, as $1 / \omega$.

\section{The distribution in angle}

The angular distribution in the dominant, asymptotic mode is of particular interest. In the notation of Eq. (44) that quantity, $F_{\infty}(x, \phi)$, is

$$
F_{\infty}(x, \phi)=\frac{i}{D_{0}} e^{i k_{0} x} \Phi_{0}(\phi)
$$

with

$$
\Phi_{0}(\phi)=\frac{1}{2 \pi} \int_{0}^{\infty} \mathrm{d} t e^{-t} \exp \left(i\left(\frac{k_{0}}{\omega}\right)[\sin \phi-\sin (\phi+\omega t)]\right)
$$

for unit source. The transient modes have a similar appearance. One of the many possible rearrangements brings us to the convenient form,

$$
\Phi_{0}(\phi)=\frac{1}{2 \pi \omega} \frac{1}{1-e^{-2 \pi / \omega}} e^{(1 / \omega) \mathcal{F}(\phi)} \int_{\phi}^{\phi+2 \pi} \mathrm{d} \alpha e^{-(1 / \omega) \mathcal{F}(\alpha)}
$$

with $\mathcal{F}(\phi)=\phi-\eta_{0} \sin \phi\left[k_{0}=i \eta_{0}(\omega, c)\right]$. And there is always the differential equation

$$
-\omega \frac{\partial}{\partial \phi} z(\phi)+\left(1-\eta_{0} \cos \phi\right) z(\phi)=\frac{1}{2 \pi}
$$

which, when solved (numerically) under the condition that $z(\phi)=z(\phi+2 \pi)$, yields a function proportional to $\Phi_{0}(\phi)$.

Once again, the question of $\eta_{0}<1$ or $\eta_{0}>1$ enters. In the former case $\mathcal{F}(\phi)$ is positive and increasing, in the latter, not so, and one encounters more dramatic behavior. Analytical information 

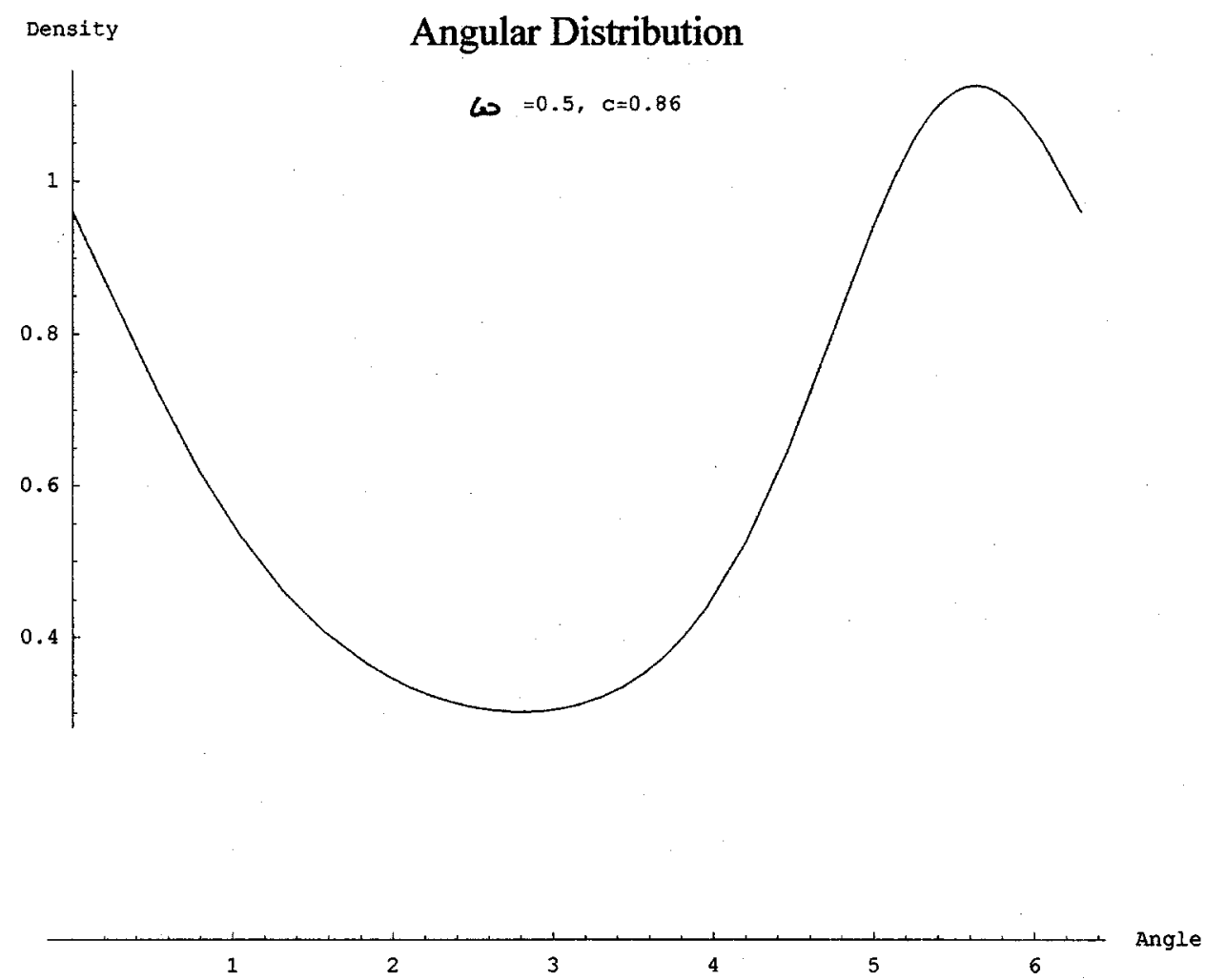

FIG. 3. Asymptotic angular distribution (2D) for $\omega=0.5, c=0.86$. Arbitrary normalization. Capture moderate, field strong.

is available when $\omega$ is small, when familiar ideas from "asymptotics" enter. ${ }^{12}$ For example, as $\omega \rightarrow 0$ the dominant contribution from the integral in Eq. (48) comes from the interval where $\mathcal{F}(\alpha)$ is minimum. In the case $\eta_{0}<1$ the minimum is at $\alpha=\phi$ and expansion about that (edge-) value yields

$$
\Phi_{0}(\phi)=\frac{1}{2 \pi} \frac{1}{\left(1-\eta_{0} \cos \phi\right)}\left[1-\omega \frac{\eta_{0} \sin \phi}{\left(1-\eta_{0} \cos \phi\right)^{2}}+\cdots\right],
$$

precisely the "outer expansion" of the solution to the differential equation. Since the outer solution satisfies the boundary condition, there is no need for a boundary layer. This simple expression displays an interesting feature of the angular distribution-a small peak centered at a small angle. The disturbance, vanishing with $\omega$, generates the transverse current.

When $\eta_{0}>1$ the situation is different. $\mathcal{F}(\alpha)$ is oscillatory, and has a single minimum, at 0 $\leqslant \phi_{*}<\pi / 2$ where $\cos \phi_{*}=\left(1 / \eta_{0}\right)$. In a subinterval of $(-\pi<\phi \leqslant \pi)$, namely, $\left(\phi_{* *}<0<\phi\right.$ $\left.<\phi_{*}\right)$, where $\mathcal{F}\left(\phi_{* *}\right)=\mathcal{F}\left(\phi_{*}\right)$, the minimum lies inside the integration of Eq. (48) and produces boundary layer behavior. $\Phi(\phi)$ rises rapidly, proportional to $\exp \left[(1 / \omega)\left(\mathcal{F}(\phi)-\mathcal{F}\left(\phi_{*}\right)\right)\right]$ then falls and passes to the nonexponential "outer"-behavior for the remainder of the interval. These features are displayed in Figs. 3 and 4. Clearly, these angular distributions are not represented well by an expansion-in-angle that is near-isotropic. The distributions associated with $\eta_{0}>1$ become quite singular in the limit of vanishing field-the continuum limit.

\section{E. Transport in three dimensions (3D)}

Since the key features are captured in the 2D case, we treat 3D briefly. We remain with plane symmetry. Then, the modifications are relatively minor. It is convenient to use two sets of angle variables. In one, the $x$ axis is the polar axis and the polar and azimuthal angles are denoted $(\theta, \psi)$; 


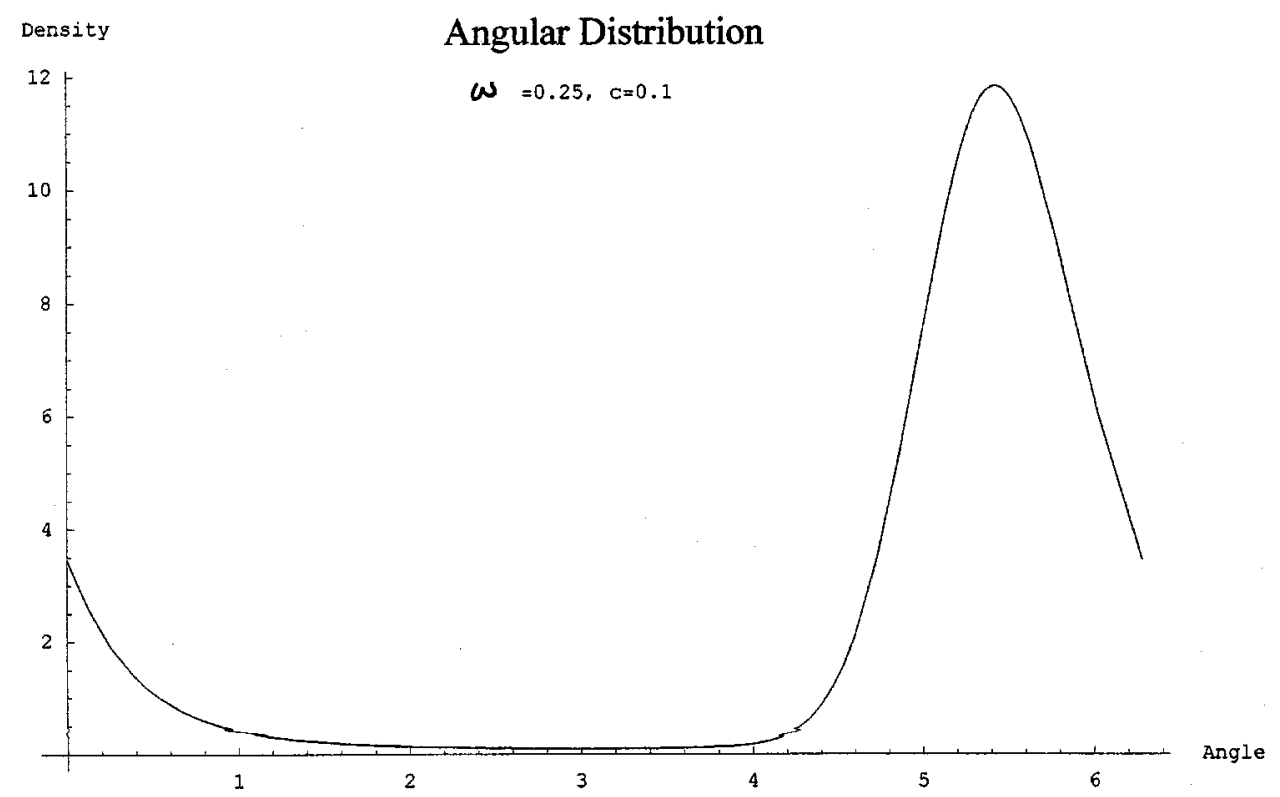

FIG. 4. Asymptotic angular distribution (2D) for $\omega=0.25, c=0.1$. Arbitrary normalization. Capture very strong, field moderate.

in the other, the $z$ axis, to which the field is parallel, is the polar axis and the angles are $(\chi, \phi)$. The streaming portion of the transport equation is altered only in that " $\mu=\cos \phi$ " is replaced by $\mu=\sin \chi \cos \phi$. Since $\chi$ enters the equation only "parametrically," it may be absorbed through much of the subsequent calculation by simply replacing the Fourier transform variable " $k$ " by $k_{\perp}=k \sin \chi$. The density is now a function of two angles, $(\chi, \phi)$. The in-scattering term is altered through the replacement,

$$
\frac{1}{2 \pi} \int_{0}^{2 \pi} \mathrm{d} \phi \rightarrow \frac{1}{4 \pi} \int \mathrm{d} \Omega=\frac{1}{4 \pi} \int_{0}^{2 \pi} \mathrm{d} \phi \int_{0}^{\pi} \mathrm{d} \chi \sin \chi
$$

The vector space is now a space of functions defined on the unit sphere, and we may use $(\theta, \psi)$ or $(\chi, \phi)$ in place of the variable, $\phi$. The inner product is now

$$
\langle g \mid f\rangle \equiv \int \mathrm{d} \Omega g^{*}(\Omega) f(\Omega)=\langle f \mid g\rangle^{*}
$$

and the ubiquitous

$$
\langle\phi \mid a\rangle=\frac{1}{\sqrt{ } 2 \pi}, \quad\langle a \mid f\rangle=\int_{0}^{2 \pi} \mathrm{d} \phi \frac{1}{\sqrt{ } 2 \pi} f(\phi)
$$

become

$$
\langle\Omega \mid a\rangle=\frac{1}{\sqrt{ } 4 \pi}, \quad\langle a \mid f\rangle=\int \mathrm{d} \Omega \frac{1}{\sqrt{ } 4 \pi} f(\Omega) .
$$

With these reinterpretations, most of the equations of the 2D case may be carried over, easily, to 3D, turning Bessel functions into spherical Bessel functions. We begin with the kernel, 
$\mathrm{K} 3$

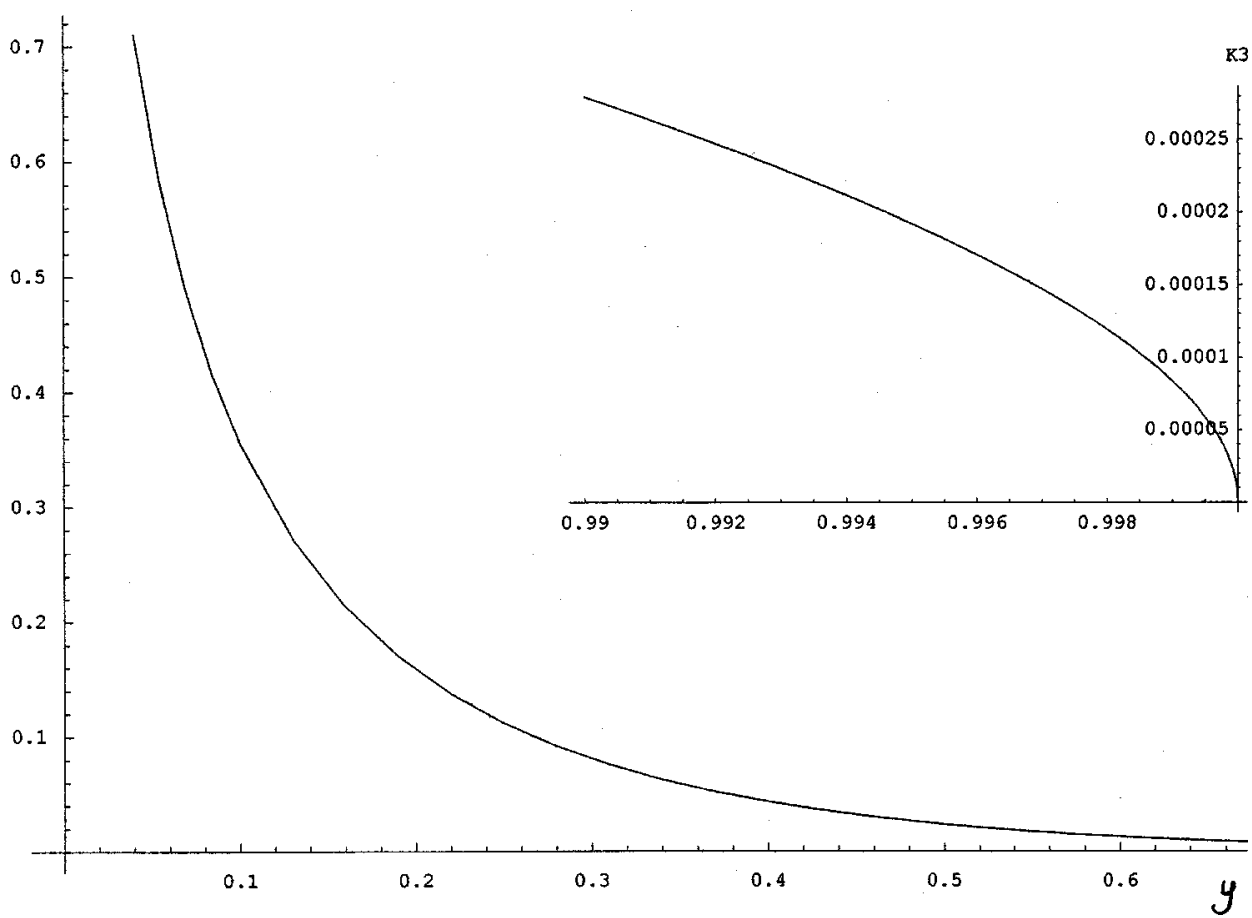

FIG. 5. $\mathcal{K}_{3}$, the 3D kernel (Peierls) for the integral equation for density, $n(x)$, with $\omega=0.5$, shown as a function of its argument, $y=\omega|x| / 2$. The kernel is zero when $y \geqslant 1$.

$$
\begin{aligned}
\left\langle a\left|\frac{1}{\mathcal{O}}\right| a\right\rangle & \rightarrow \frac{1}{4 \pi} \int_{0}^{\infty} \mathrm{d} t e^{-t} \int \mathrm{d} \Omega e^{i \kappa_{\perp}[\sin \phi-\sin (\phi+\omega t)]} \\
& =\int_{0}^{\infty} \mathrm{d} t e^{-t} \frac{1}{2} \int_{0}^{\pi} \mathrm{d} \chi \sin \chi J_{0}\left(2 \kappa \sin \frac{\omega t}{2} \sin \chi\right) .
\end{aligned}
$$

Here, and in the discussion of currents, the relation

$$
\int_{0}^{\pi / 2} \mathrm{~d} \chi \sin ^{n+1} \chi J_{n}(z \sin \chi)=j_{n}(z)
$$

between ordinary and spherical Bessel functions is useful. Then,

$$
\mathcal{K}_{3}(k, \omega) \equiv\left\langle a\left|\frac{1}{\mathcal{O}}\right| a\right\rangle=\int_{0}^{\infty} \mathrm{d} t e^{-t} j_{0}\left(2 \kappa \sin \frac{\omega t}{2}\right)=\frac{2}{\omega} \frac{1}{\sinh \left(\frac{\pi}{\omega}\right)} \int_{0}^{\pi / 2} \mathrm{~d} \phi \cosh \left(\frac{2 \phi}{\omega}\right) j_{0}\left(2 \frac{k}{\omega} \cos \phi\right),
$$

with $j_{0}(z)=\sin z / z$.

Upon expansion to produce a power series in $k$, one finds

$$
\mathcal{K}_{3}(k, \omega)=\left[1+\sum_{m=1}^{\infty} \frac{\left(-k^{2}\right)^{m}}{2 m+1} \prod_{r=1}^{m} \frac{1}{1+r^{2} \omega^{2}}\right] .
$$


Setting $\omega=0$ gives the familiar logarithm of the field-free case, and expanding for small $\omega$ in the manner given earlier [e.g., Eqs. (27) and (37)] yields the singular and asymptotic sequence

$$
\mathcal{K}_{3}(k, \omega)=\frac{1}{2 i k} \log \frac{1+i k}{1-i k}+\frac{1}{3} \omega^{2} \frac{k^{2}}{\left(k^{2}+1\right)^{3}}+\cdots+\text { exponentially small terms. }
$$

The regime $\omega \gg 1$ is dealt with best via the series, Eq. (49), giving

$$
\mathcal{K}_{3}(k, \omega) \asymp \frac{\omega}{2 k} \int_{0}^{2 k / \omega} \mathrm{d} z J_{0}(z)
$$

These expressions are helpful in determining the zeros of $\mathcal{D}_{3}(k, \omega, c)$, which, like its predecessor, is entire-in- $k$. The behavior of these zeros is quite similar to their $2 \mathrm{D}$ counterparts. Turning to the picture in $x$ coordinates, we note that the Peierls kernel may be obtained by Fourier-inverting Eq. (49) to get a pretty, "3-Sine" formula for the even function,

$$
\mathcal{K}_{3}\left(x-x^{\prime}, \omega\right)=\frac{1}{1-e^{-4 \pi / \omega}} \int_{0}^{2 \pi} \mathrm{d} \theta \int \mathrm{d} \Omega e^{-2 \theta / \omega} \delta\left(\frac{\omega}{2}\left(x-x^{\prime}\right)-\sin \theta \sin \phi \sin \chi\right) .
$$

Comments made earlier about its compact support continue to hold. On the other hand, we may Fourier-invert Eq. (50) to get quite a different compact form,

$$
\mathcal{K}_{3}(x, \omega)=\frac{1}{2 \sinh \left(\frac{\pi}{\omega}\right)} \int_{0}^{\phi_{*}(\omega x)} \frac{\mathrm{d} \phi}{\cos \phi} \cosh \frac{2 \phi}{\omega}
$$

( $\cos \phi_{*}=(\omega / 2) x$, and $\left.|(\omega / 2) x|<1\right)$. In fact, $\mathcal{K}_{3}$ may be shown to vanish with vertical tangent. (See Fig. 5.) That this rather peculiar expression does become the familiar exponential integral when $\omega \rightarrow 0$ may be seen by setting

$$
\phi=\left(\frac{\pi}{2}-\psi\right)
$$

expanding the cosh, and passing to the limit.

Currents. We have, generally,

$$
\mathcal{J}_{x, y, z}(k) \equiv \mathcal{J}(k)=\left\langle j\left|\frac{1}{\mathcal{O}}\right| Q\right\rangle+\frac{c}{\mathcal{D}(k, c)}\left\langle j\left|\frac{1}{\mathcal{O}}\right| a\right\rangle\left\langle a\left|\frac{1}{\mathcal{O}}\right| Q\right\rangle .
$$

When the source is isotropic, and normalized to $\sqrt{ } 4 \pi$,

$$
\mathcal{J}(k)=\frac{1}{\mathcal{D}(k, c)}\left\langle j\left|\frac{1}{\mathcal{O}}\right| a\right\rangle
$$

and we discuss the numerator of this expression,

$$
\frac{1}{\sqrt{4 \pi}}\left\langle j_{x, y}\left|\frac{1}{\mathcal{O}}\right| a\right\rangle=\frac{1}{4 \pi} \int_{0}^{\infty} \mathrm{d} t e^{-t} \int \mathrm{d} \Omega \sin \chi[\cos \phi, \sin \phi] e^{i \kappa_{\perp}[\sin \phi-\sin (\phi+\omega t)]},
$$




$$
\begin{aligned}
\frac{1}{\sqrt{4 \pi}}\left\langle j_{z}\left|\frac{1}{\mathcal{O}}\right| a\right\rangle & =\frac{1}{4 \pi} \int_{0}^{\infty} \mathrm{d} t e^{-t} \int \mathrm{d} \Omega \cos \chi e^{i \kappa_{\perp}[\sin \phi-\sin (\phi+\omega t)]} \\
& =\int_{0}^{\infty} \mathrm{d} t e^{-t} \frac{1}{2} \int_{0}^{\pi} \mathrm{d} \chi \sin \chi \cos \chi J_{0}\left(2 \kappa \sin \chi \sin \frac{\omega t}{2}\right)=0, \quad \text { by symmetry. }
\end{aligned}
$$

Thus, quite generally,

$$
\mathcal{J}_{z}(k)=\left\langle j_{z}\left|\frac{1}{\mathcal{O}}\right| Q\right\rangle
$$

an expression which is, at first sight, puzzling, for the current appears to be independent of capture. A moment's thought convinces one of its correctness, and that $J_{z}$ is, in any case, trivial. We merely note that with isotropic scattering, a beam becomes distributed isotropically at the first collision. After that collision, only a density gradient will drive a current. But in our problem, there are no gradients in the $z$ direction; $J_{z}$ exists only in the interval from birth to first collision when the value of " $c$ " is irrelevant. This argument holds for particles immersed in an arbitrary external field that is independent of $z$. The $z$ current is carried only by uncollided particles. Adding a bit of anisotropy to the scattering changes the result significantly.

The current components, $\mathcal{J}_{x, y}(k)$, are simply the weighted $\chi$ average of their counterparts in two dimensions, Eq. (39). The connection between ordinary and spherical Bessel functions then gives the concise result,

$$
\begin{aligned}
& i \mathcal{J}_{x}(k)=\frac{1}{\mathcal{D}_{3}(k, c)} \int_{0}^{\infty} \mathrm{d} t e^{-t} \cos \frac{\omega t}{2} j_{1}\left(2 \kappa \sin \frac{\omega t}{2}\right), \\
& -i \mathcal{J}_{y}(k)=\frac{1}{\mathcal{D}_{3}(k, c)} \int_{0}^{\infty} \mathrm{d} t e^{-t} \sin \frac{\omega t}{2} j_{1}\left(2 \kappa \sin \frac{\omega t}{2}\right),
\end{aligned}
$$

for currents in three dimensions.

Angular distributions. Expressions for the angular distribution associated with dominant and transient modes may be obtained easily. One simply replaces $k_{0}$ with $k_{0} \sin \chi$ in Eq. (47). The distributions are then symmetric with respect to the $(x-y)$ plane and fixing a value of $\chi$ is equivalent to selecting one of the 2D distributions we have described earlier. As $\chi$ decreases from its in-plane value of $\pi / 2$, the effective $\eta_{0}$ diminishes and the corresponding distribution is smoother. Overall, angular distributions in 3D appear to be smoother than those in 2D.

One can proceed further with details of the $3 \mathrm{D}$ case, in a manner similar to that of $2 \mathrm{D}$ but it is clear that the $2 \mathrm{D}$ case displays almost everything that is interesting about the problem. The next step should be an attack upon the time-dependent problem, which is simple enough after Laplacetransform, but whose inversion is a complicated matter.

\section{ACKNOWLEDGMENTS}

The author is grateful for a friendly and helpful correspondence with Professor R. E Robson and Professor K. Kumar, during the early phase of this research.

\section{APPENDIX}

An alternative, easier way to evaluate the key quantity,

$$
\left\langle a\left|\frac{1}{\mathcal{O}}\right| a\right\rangle=\frac{1}{2 \pi} \int_{0}^{\infty} \mathrm{d} t e^{-t} \int_{0}^{2 \pi} \mathrm{d} \phi e^{i \kappa[\sin \phi-\sin (\phi+\omega t)]}
$$


is to notice that $[\sin \phi-\sin (\phi+\omega t)]=-2 \sin (\omega t / 2) \cos (\phi+\omega t / 2)$, and that the replacement of $(\phi$ $+\omega t / 2)$ by $\phi$ does not alter the value of the integral. The expression

$$
\left\langle a\left|\frac{1}{\mathcal{O}}\right| a\right\rangle=\int_{0}^{\infty} \mathrm{d} t e^{-t} J_{0}\left(2 \kappa \sin \frac{\omega t}{2}\right)
$$

follows at once. In fact, evaluation of the matrix element in the expression for the current induced by an isotropic source

$$
\begin{gathered}
J_{x, y}(k)=\frac{1}{\mathcal{D}(k)}\left\langle j_{x, y}\left|\frac{1}{\mathcal{O}}\right| a\right\rangle, \\
\frac{1}{\sqrt{2 \pi}}\left\langle j_{x, y}\left|\frac{1}{\mathcal{O}}\right| a\right\rangle=\frac{1}{2 \pi} \int_{0}^{\infty} \mathrm{d} t e^{-t} \int_{0}^{2 \pi} \mathrm{d} \phi[\cos \phi, \sin \phi] e^{i \kappa[\sin \phi-\sin (\phi+\omega t)]}
\end{gathered}
$$

is made easy by the same approach. The change of variable $(\phi+\omega t / 2) \rightarrow \phi$ and integration by parts produces the results noted earlier.

${ }^{1}$ L. G. H. Huxley and R. W. Crompton, The Diffusion and Drift of Electrons in Gases (Wiley-Interscience, New York, 1974).

${ }^{2}$ E. A. Mason and E. W. McDaniel, Transport Properties of Ions in Gases (Wiley, New York, 1988).

${ }^{3}$ K. Kumar, H. R. Skullerud, and R. E. Robson, Aust. J. Phys. 33, 343 (1980).

${ }^{4}$ K. Kumar, Phys. Rep. 112, 319 (1984).

${ }^{5}$ R. E. Robson, R. Winkler, and F. Sigeneger, Phys. Rev. E 65, 056410 (2002).

${ }^{6}$ K. F. Ness, Phys. Rev. E 47, 327 (1993).

${ }^{7}$ K. M. Case and P. F. Zweifel, Linear Transport Theory (Addison-Wesley, Reading, MA, 1967).

${ }^{8}$ See, for example, F. F. Chen, Introduction to Plasma Physics (Plenum, New York, 1974).

${ }^{9}$ H. Grad, Commun. Pure Appl. Math. 14, 323 (1961); Handbuch der Physik (Springer, Berlin, 1958), Vol. 12.

${ }^{10}$ A. V. Bobylev, A. Hansen, E. H. Hauge, F. A. Maaø, and J. Piasecki, Phys. Rev. Lett. 75, 197 (1995); J. Stat. Phys. 87, 1205 (1997); 102, 1133 (2001).

${ }^{11}$ R. E. Peierls, Proc. Cambridge Philos. Soc. 35, 610 (1939).

${ }^{12}$ C. M. Bender and S. A. Orszag, Advanced Mathematical Methods for Scientists and Engineers (McGraw-Hill, New York, 1978). 